\title{
Setting standard scores for acceptance tests in Sport schools and its relationship with body mass index Dr/ Hani Mohammed Zkria Azab ${ }^{(*)}$
}

\section{Introduction:}

Interest in school sport is the basis for progress sports level in general. Measurements and selection is the first step towards achieving this progress.

\section{School}

physical education is an integral part of educational system as a whole; it contributes to individuals' growth and maturity through motor and physical experiences. (Allawi, \& Elshafee, 2000 p207)

Tests in sports field have several and different functions and uses including future performance prediction; which mean future forecasting of specific results in light of measurements made. In light of motor and physical ability of student or player measured at a certain time it is possible to predict its level or a related ability level in future time. (Radwan, 2006, p. 47)

Standards settings have international interest, where standards is applicable in many educational systems in developed and developing countries as well. Preparation of Arabic education national standard levels is a need for education reform in Egypt. (Ministry of Education, 2003, p.3)

Standard settings is important as it may guide the teacher or coach to evaluate whether athletes or individuals scores in mid-level, aboveaverage or less than average for standardizing sample used in developing the standards. (Allawi, \& Radwan, 2000, p. 381)

Hassanien (2002, p. 154) explains that standards allows the individual taking the tests to recognize his relative position in the group, and this is an important and necessary action to achieve ideal evaluation, it identifies the individual position and contribute in setting total marks for various units in

\footnotetext{
Lecturer, department of educational, psychological and social sciences - Faculty of Physical Education for Men-Benha University Assiut Journal For Sport Science Arts
} 
specific measurement units in physical performance.

Some scientific studies indicate the importance of investigating relationship between anthropometric measurements and physical fitness using multiple analysis methods. (Maciaszek, \& Osiński 2001).

The researcher believes that any test standards are always temporary standards؛ it will be invalid for comparison with time due to individuals' abilities, characteristics and traits changing over the years. Therefore, test standards should be reconsidered from time to time due to continued progress in teachers and trainers preparation and teaching and training methods.

The researcher as a member in Qaliubiya sport school Board of Trustees and by attending its admission tests noted that these admission tests since school started in the academic year 1994/1995 is performed as per tests prepared by Ministry of education. These tests have no standard scores (Z, T, or percentile), the matter which makes random students evaluation without any scientific or reference basis, not allowing individual self-evaluation and comparing with peers to determine his relative level in the sample. Therefore, choosing accepted students may not be right and make some competent students miss the opportunity, or giving an opportunity for those who do not deserve it. At the start of practical training in the school, specialists trainer surprise that there are some students who are enrolled in school do not have the minimum physical fitness that allow their practice specialized sport in different games inside the school. Respectively, errors accumulate as the student who inaccurately took others opportunity will continue in the school for 6 years (preparatory - secondary school period). The Ministerial Resolution No. 325 on 03/09/2013 and General Education law have nothing to allow exclusion of student chosen by mistake. It is noteworthy to mention that this is not intentionally or deliberately, but because of not following scientific method and approach in converting raw scores derived from tests to standard scores to evaluate student performance. 
After the end of school admission tests the five members Committee met (Directorate general guider, senior area guider, school director, and two vicedirectors) to express an opinion on scoring mechanism and determine maximum score for each test separately.

It is not reasonable in the third millennium, with different sciences intertwined with each other to grow and live up the whole humanity, including physical education and sport science that students' admission tests sports school carried in this primitive and indiscriminate method. This method make nothing other than losing of opportunities to distinguished students and make usefulness efforts with other students without and waste public money in expending on those students represented in disbursement of bonuses for trainers and supervisors and committees in charge for their work.

In the end, the sport school it does not achieve its objective in preparation of skill and physical privileged player may be in one-day an international or Olympia player represents national teams in various games.

The sports school have no standard scores of physical characteristics of admission applicants, and this is what called the researcher to try to set standard scores of physical abilities and find out the ability to predict physical ability knowing body mass index.

\section{The research aims:}

1- Setting standard scores for physical abilities (speed, agility, legs power, arms power, and abdominal muscles power) and the total standard score for acceptance in Qaliubiya sports school in 2012-2013 to 2014-2015

2- Predict physical abilities level using body mass index.

\section{Research Queries:}

1- What are the standard scores for physical abilities (speed, agility, legs power, arms power, and abdominal muscles power) and the total standard score for acceptance in Qaliubiya sports school in 2012-2013 to 2014-2015?

2- Is it possible to predict physical abilities level using body mass index.

\section{Research definitions Standard}

The criteria measure learner expected performance; 
it takes two directions, namely the minimum behavior and the generality in determining performance level. (Homs, 1997, p. 52)

\section{Body Mass Index (BMI:}

It is a global scale to determine the degree of obesity؛ it calculated as body weight $(\mathrm{kg})$ divided by the square of body height (m). It recognized as best obesity measure by US National Health institution and the World Health Organization (Visscher,, Snijder \& ،Seidell, 2010, p .5)

\section{Standard level:}

It is the standard criteria used to determine the relative state of raw scores for the purpose of score interpretation evaluate the results. (Allawi \& Radwan, 2000, p. 194)

\section{Previous studies}

\section{Abo Shady, Abdulsalam, \&} Mohamed (2005) study entitled "Setting standard levels for some physical and physiological variables in late childhood" and aimed at determine standard levels to use in physical and physiological measurements evaluation of pupils in late childhood in Assiut governorate. approach used. Sample included (599) child. The most important results were availability of positive correlation between physical and physiological variables, for pupils in late childhood in Assiut governorate.

Saleh (2013) study entitled Setting standard levels for "Physical fitness and some anthropometric measurements for higher basic education stage in Gonain directorate". The study aimed at set standard levels for some physical fitness elements and some anthropometric measurements for female students in high basic education stage. Descriptive approach used. Sample included (1066) Student. The most important findings was setting the required standard levels.

Mohamed (2007) study entitled "Setting standard levels for some physical and skill characteristics for basketball juniors (14-16 years)". Study aimed at set standards for some physical components for basketball juniors under 16 years. Descriptive approach used. Sample included (64) basketball junior. The most important results were setting the required standard scores. 
Research procedures

Research Methodology:

Descriptive approach used because of its appropriateness of research nature.

Research sample:
Research sample consisted of (640) pupil addition applicants to Qaliubiya governorate preparatory sport school for academic years from 20122013 to 2014-2015, i.e. three

Table (1) years applicants.

Descriptive statistics for research sample $(n=640)$

\begin{tabular}{|c|c|c|c|c|c|}
\hline No. & variables & $\begin{array}{c}\text { measurement } \\
\text { unit }\end{array}$ & average & $\begin{array}{l}\text { Standard } \\
\text { deviation }\end{array}$ & skewness \\
\hline 1 & Height & Meter & 148.31 & 7.37 & 0.22 \\
\hline 2 & weight & $\mathrm{Kg}$ & 44.09 & 10.65 & 1.40 \\
\hline 3 & BMI & - & 19.86 & 3.54 & 1.37 \\
\hline 4 & $\begin{array}{l}\text { speed ( } 30 \mathrm{~m} \\
\text { running) }\end{array}$ & Meter & 6.30 & 1.03 & 0.18 \\
\hline 5 & agility & Meter & 8.94 & 0.67 & 0.77 \\
\hline 6 & Legs power & second & 1.61 & 0.20 & $0.02-$ \\
\hline 7 & Arms power & second & 3.72 & 1.83 & 0.93 \\
\hline 8 & $\begin{array}{l}\text { abdomen } \\
\text { muscles } \\
\text { strength }\end{array}$ & number & 23.97 & 3.81 & 0.07 \\
\hline $\begin{array}{l}\text { that } \\
\text { vari } \\
\text { and } \\
\text { matt } \\
\text { norn } \\
\text { vari } \\
\text { Stat }\end{array}$ & $\begin{array}{l}\text { Table (1) } \mathrm{r} \\
\text { skewness fac } \\
\text { bles ranged } \\
\text { l.40) i.e. betw } \\
\text { er which indi } \\
\text { lality distribut } \\
\text { bles. } \\
\text { stical process } \\
\text { The resea }\end{array}$ & $\begin{array}{l}\text { ults reveal } \\
\text { rs for all } \\
\text { tween }(-2 \\
\text { ( } \pm 3) \text {, the } \\
\text { tes sample } \\
\text { in these } \\
\text { g: } \\
\text { her used }\end{array}$ & \multicolumn{3}{|c|}{$\begin{array}{l}\text { Mean , standard } \\
\text { deviation, T standard score, } \\
\text { and Percentile standard score } \\
\text { Research results: } \\
\text { T scores and percentile scores } \\
\text { obtained from data processing } \\
\text { in SPSS for the five physical } \\
\text { abilities tests represented in } \\
\text { tables (2) to (6) }\end{array}$} \\
\hline
\end{tabular}

SPSS statistical program to extract the following:

Table (2) 
T standard score and percentile standard score for speed test for applicant students for addition in Qaliubiya governorate sport $\operatorname{school}(n=640)$

\begin{tabular}{|c|c|c|c|c|c|c|c|c|c|c|c|}
\hline No & $\begin{array}{l}\text { Raw } \\
\text { score }\end{array}$ & $\begin{array}{c}\mathbf{T} \\
\text { score }\end{array}$ & $\begin{array}{c}\text { Percentile } \\
\text { score }\end{array}$ & No & $\begin{array}{l}\text { Raw } \\
\text { score }\end{array}$ & $\begin{array}{c}\mathbf{T} \\
\text { score }\end{array}$ & $\begin{array}{c}\text { Percentile } \\
\text { score }\end{array}$ & No & $\begin{array}{l}\text { Raw } \\
\text { score }\end{array}$ & $\begin{array}{c}\mathbf{T} \\
\text { score }\end{array}$ & $\begin{array}{c}\text { Percentile } \\
\text { score }\end{array}$ \\
\hline 1. & 10.03 & 29.85 & 0.16 & 26. & 7.98 & 36.63 & 8.59 & 51. & 7.44 & 39.44 & 21.02 \\
\hline 2. & 9.06 & 31.88 & 0.31 & 27. & 7.97 & 36.73 & 9.14 & 52. & 7.43 & 39.54 & 21.80 \\
\hline 3. & 9.01 & 32.66 & 0.47 & 28. & 7.96 & 36.82 & 9.53 & 53. & 7.42 & 39.63 & 22.50 \\
\hline 4. & 8.72 & 32.85 & 0.63 & 29. & 7.92 & 36.92 & 9.84 & 54. & 7.41 & 39.73 & 23.05 \\
\hline 5. & 8.60 & 33.72 & 0.78 & 30. & 7.83 & 37.02 & 10.31 & 55. & 7.40 & 39.83 & 23.67 \\
\hline 6. & 8.57 & 33.92 & 1.02 & 31. & 7.74 & 37.41 & 11.25 & 56. & 7.39 & 39.92 & 24.14 \\
\hline 7. & 8.56 & 34.01 & 1.25 & 32. & 7.73 & 37.50 & 12.11 & 57. & 7.38 & 40.12 & 24.45 \\
\hline 8. & 8.54 & 34.40 & 1.48 & 33. & 7.70 & 37.60 & 12.42 & 58. & 7.36 & 40.21 & 24.69 \\
\hline 9. & 8.50 & 34.69 & 1.80 & 34. & 7.68 & 37.70 & 12.81 & 59. & 7.33 & 40.41 & 25.00 \\
\hline 10. & 8.44 & 34.79 & 2.03 & 35. & 7.67 & 37.79 & 13.20 & 60. & 7.31 & 40.51 & 25.31 \\
\hline 11. & 8.43 & 34.98 & 2.27 & 36. & 7.66 & 37.99 & 13.67 & 61. & 7.30 & 40.60 & 25.70 \\
\hline 12. & 8.39 & 35.08 & 2.58 & 37. & 7.65 & 38.08 & 14.45 & 62. & 7.29 & 40.70 & 26.17 \\
\hline 13. & 8.38 & 35.18 & 2.89 & 38. & 7.63 & 38.18 & 15.08 & 63. & 7.28 & 40.80 & 26.56 \\
\hline 14. & 8.36 & 35.27 & 3.28 & 39. & 7.62 & 38.28 & 15.47 & 64. & 7.27 & 40.89 & 27.11 \\
\hline 15. & 8.35 & 35.37 & 3.67 & 40. & 7.57 & 38.37 & 16.09 & 65. & 7.26 & 40.99 & 27.66 \\
\hline 16. & 8.31 & 35.47 & 4.06 & 41. & 7.55 & 38.66 & 16.64 & 66. & 7.25 & 41.09 & 28.20 \\
\hline 17. & 8.30 & 35.56 & 4.38 & 42. & 7.54 & 38.76 & 17.03 & 67. & 7.23 & 41.28 & 28.67 \\
\hline 18. & 8.26 & 35.66 & 4.84 & 43. & 7.53 & 38.86 & 17.66 & 68. & 7.21 & 41.38 & 28.91 \\
\hline 19. & 8.24 & 35.76 & 5.39 & 44. & 7.52 & 38.96 & 18.44 & 69. & 7.20 & 41.47 & 29.14 \\
\hline 20. & 8.20 & 35.95 & 5.63 & 45. & 7.51 & 39.05 & 19.14 & 70. & 7.19 & 41.57 & 29.38 \\
\hline 21. & 8.10 & 36.05 & 5.86 & 46. & 7.50 & 39.15 & 19.53 & 71. & 7.18 & 41.67 & 29.53 \\
\hline 22. & 8.06 & 36.15 & 6.17 & 47. & 7.49 & 39.25 & 19.84 & 72. & 7.17 & 41.76 & 29.92 \\
\hline 23. & 8.04 & 36.34 & 6.72 & 48. & 7.48 & 39.25 & 19.84 & 73. & 7.16 & 41.86 & 30.55 \\
\hline 24. & 8.02 & 36.44 & 7.50 & 49. & 7.46 & 39.25 & 19.84 & 74. & 7.15 & 41.96 & 30.94 \\
\hline 25. & 8.00 & 36.53 & 8.13 & 50. & 7.45 & 39.34 & 20.39 & 75. & 7.14 & 42.06 & 31.09 \\
\hline
\end{tabular}

Table (2) (Cont.)

T standard score and percentile standard score for speed test for applicant students for addition in Qaliubiya governorate sport $\operatorname{school}(n=640)$

\begin{tabular}{c|c|c|c|c|c|c|c|c|c|c|c}
\hline \hline No & $\begin{array}{c}\text { Raw } \\
\text { score }\end{array}$ & $\begin{array}{c}\text { T } \\
\text { score }\end{array}$ & $\begin{array}{c}\text { Percentile } \\
\text { score }\end{array}$ & No & $\begin{array}{c}\text { Raw } \\
\text { score }\end{array}$ & $\begin{array}{c}\text { T } \\
\text { score }\end{array}$ & $\begin{array}{c}\text { Percentile } \\
\text { score }\end{array}$ & No & $\begin{array}{c}\text { Raw } \\
\text { score }\end{array}$ & $\begin{array}{c}\text { T } \\
\text { score }\end{array}$ & $\begin{array}{c}\text { Percentile } \\
\text { score }\end{array}$ \\
\hline \hline 76. & 7.13 & 42.15 & 31.48 & 107. & 6.77 & 46.71 & 40.94 & 138. & 6.41 & 51.36 & 48.59 \\
\hline 77. & 7.12 & 42.35 & 31.95 & 108. & 6.76 & 46.80 & 41.09 & 139 & 6.40 & 51.74 & 49.06 \\
\hline 78. & 7.11 & 42.44 & 32.50 & 109. & 6.75 & 47.00 & 41.25 & 140. & 6.39 & 51.84 & 49.61 \\
\hline 79. & 7.10 & 42.54 & 33.05 & 110. & 6.74 & 47.19 & 41.41 & 141. & 6.38 & 51.94 & 50.31 \\
\hline 80. & 7.09 & 42.64 & 33.52 & 111. & 6.73 & 47.29 & 41.56 & 142. & 6.36 & 52.03 & 50.94 \\
\hline 81. & 7.07 & 42.73 & 33.91 & 112. & 6.72 & 47.67 & 41.72 & 143. & 6.35 & 52.13 & 51.09 \\
\hline 82. & 7.05 & 42.83 & 34.30 & 113. & 6.71 & 47.96 & 41.88 & 144. & 6.34 & 52.23 & 51.25 \\
\hline \hline
\end{tabular}


Follow Table (2) (Cont.)

T standard score and percentile standard score for speed test for applicant students for addition in Qaliubiya governorate sport $\operatorname{school}(n=640)$

\begin{tabular}{|c|c|c|c|c|c|c|c|c|c|c|c|}
\hline No & $\begin{array}{l}\text { Raw } \\
\text { score }\end{array}$ & $\begin{array}{c}\mathrm{T} \\
\text { score }\end{array}$ & $\begin{array}{c}\text { Percentile } \\
\text { score }\end{array}$ & No & $\begin{array}{l}\text { Raw } \\
\text { score }\end{array}$ & $\begin{array}{c}\mathrm{T} \\
\text { score }\end{array}$ & $\begin{array}{c}\text { Percentile } \\
\text { score }\end{array}$ & No & $\begin{array}{l}\text { Raw } \\
\text { score }\end{array}$ & $\begin{array}{c}\mathrm{T} \\
\text { score }\end{array}$ & $\begin{array}{c}\text { Percentile } \\
\text { score }\end{array}$ \\
\hline 83. & 7.02 & 42.93 & 34.69 & 114. & 6.70 & 48.06 & 42.27 & 145. & 6.33 & 52.32 & 51.64 \\
\hline 84. & 7.01 & 43.02 & 35.00 & 115. & 6.69 & 48.16 & 42.66 & 146. & 6.32 & 52.42 & 52.03 \\
\hline 85. & 7.00 & 43.12 & 35.31 & 116. & 6.68 & 48.35 & 42.81 & 147. & 6.29 & 52.52 & 52.42 \\
\hline 86. & 6.99 & 43.41 & 35.70 & 117. & 6.66 & 48.45 & 42.97 & 148. & 6.27 & 52.61 & 52.97 \\
\hline 87. & 6.98 & 43.51 & 36.17 & 118. & 6.65 & 48.55 & 43.13 & 149. & 6.26 & 52.71 & 53.52 \\
\hline 88. & 6.97 & 43.70 & 36.41 & 119. & 6.64 & 48.74 & 43.28 & 150. & 6.23 & 52.81 & 54.22 \\
\hline 89. & 6.96 & 43.80 & 36.64 & 120. & 6.63 & 48.84 & 43.52 & 151. & 6.22 & 52.91 & 54.92 \\
\hline 90. & 6.95 & 43.90 & 36.88 & 121. & 6.62 & 49.03 & 43.75 & 152. & 6.21 & 53.00 & 55.31 \\
\hline 91. & 6.94 & 43.99 & 37.03 & 122. & 6.61 & 49.13 & 43.98 & 153. & 6.20 & 53.10 & 55.70 \\
\hline 92. & 6.93 & 44.09 & 37.19 & 123. & 6.60 & 49.22 & 44.22 & 154. & 6.18 & 53.20 & 56.17 \\
\hline 93. & 6.92 & 44.19 & 37.34 & 124. & 6.59 & 49.32 & 44.45 & 155. & 6.17 & 53.29 & 56.41 \\
\hline 94. & 6.91 & 44.28 & 37.58 & 125. & 6.58 & 49.61 & 44.77 & 156. & 6.15 & 53.39 & 56.64 \\
\hline 95. & 6.90 & 44.77 & 37.81 & 126. & 6.57 & 49.71 & 45.00 & 157. & 6.14 & 53.49 & 56.95 \\
\hline 96. & 6.89 & 45.06 & 38.05 & 127. & 6.56 & 49.90 & 45.16 & 158. & 6.13 & 53.68 & 57.19 \\
\hline 97. & 6.88 & 45.16 & 38.44 & 128. & 6.55 & 50.19 & 45.39 & 159. & 6.11 & 53.78 & 57.42 \\
\hline 98. & 6.87 & 45.25 & 38.83 & 129. & 6.54 & 50.29 & 45.70 & 160. & 6.10 & 53.87 & 57.73 \\
\hline 99. & 6.85 & 45.35 & 39.22 & 130. & 6.53 & 50.39 & 45.94 & 161. & 6.09 & 53.97 & 58.36 \\
\hline 100. & 6.84 & 45.54 & 39.53 & 131. & 6.52 & 50.48 & 46.17 & 162. & 6.06 & 54.07 & 58.98 \\
\hline 101. & 6.83 & 45.64 & 39.69 & 132. & 6.51 & 50.58 & 46.56 & 163. & 6.02 & 54.16 & 59.22 \\
\hline 102. & 6.82 & 45.83 & 39.84 & 133. & 6.50 & 50.77 & 46.88 & 164. & 6.01 & 54.26 & 59.53 \\
\hline 103. & 6.81 & 46.03 & 40.16 & 134. & 6.49 & 50.87 & 47.11 & 165. & 5.99 & 54.36 & 60.00 \\
\hline 104. & 6.80 & 46.12 & 40.47 & 135. & 6.48 & 50.97 & 47.50 & 166. & 5.97 & 54.46 & 60.55 \\
\hline 105. & 6.79 & 46.41 & 40.63 & 136. & 6.44 & 51.06 & 47.97 & 167. & 5.96 & 54.55 & 61.25 \\
\hline 106. & 6.78 & 46.51 & 40.78 & 137. & 6.42 & 51.16 & 48.36 & 168. & 5.94 & 54.65 & 61.80 \\
\hline 107. & 5.93 & 54.75 & 62.19 & 200. & 5.47 & 58.23 & 78.98 & 231. & 5.15 & 61.91 & 90.78 \\
\hline 108. & 5.90 & 54.84 & 62.66 & 201. & 5.46 & 58.33 & 79.53 & 232. & 5.14 & 62.01 & 91.02 \\
\hline 109. & 5.89 & 54.94 & 63.05 & 202. & 5.45 & 58.43 & 80.23 & 233. & 5.13 & 62.11 & 91.25 \\
\hline 110. & 5.87 & 55.04 & 63.98 & 203. & 5.44 & 58.52 & 80.70 & 234. & 5.10 & 62.30 & 91.48 \\
\hline 111. & 5.85 & 55.13 & 64.92 & 204. & 5.43 & 58.62 & 80.94 & 235. & 5.09 & 62.79 & 91.72 \\
\hline 112. & 5.84 & 55.23 & 65.23 & 205. & 5.42 & 58.72 & 81.09 & 236. & 5.08 & 62.88 & 91.88 \\
\hline 113. & 5.82 & 55.33 & 65.47 & 206. & 5.41 & 58.81 & 81.33 & 237. & 5.07 & 63.08 & 92.03 \\
\hline 114. & 5.81 & 55.52 & 65.86 & 207. & 5.40 & 59.01 & 81.56 & 238. & 5.06 & 63.17 & 92.19 \\
\hline
\end{tabular}


Follow Table (2) (Cont.)

$T$ standard score and percentile standard score for speed test for applicant students for addition in Qaliubiya governorate sport $\operatorname{school}(n=640)$

\begin{tabular}{|c|c|c|c|c|c|c|c|c|c|c|c|}
\hline No & $\begin{array}{l}\text { Raw } \\
\text { score }\end{array}$ & $\begin{array}{c}\mathbf{T} \\
\text { score }\end{array}$ & $\begin{array}{c}\text { Percentile } \\
\text { score }\end{array}$ & No & $\begin{array}{l}\text { Raw } \\
\text { score }\end{array}$ & $\begin{array}{c}\mathbf{T} \\
\text { score }\end{array}$ & $\begin{array}{c}\text { Percentile } \\
\text { score }\end{array}$ & No & $\begin{array}{l}\text { Raw } \\
\text { score }\end{array}$ & $\begin{array}{c}\mathbf{T} \\
\text { score }\end{array}$ & $\begin{array}{c}\text { Percentile } \\
\text { score }\end{array}$ \\
\hline 169. & 5.80 & 55.62 & 66.41 & 208. & 5.38 & 59.20 & 81.80 & 239 . & 5.04 & 63.27 & 92.50 \\
\hline 170. & 5.79 & 55.71 & 66.88 & 209. & 5.37 & 59.30 & 82.03 & 240. & 5.03 & 63.37 & 93.05 \\
\hline 171. & 5.76 & 55.81 & 67.19 & 210. & 5.36 & 59.40 & 82.27 & 241. & 5.02 & 63.56 & 93.44 \\
\hline 172. & 5.71 & 55.91 & 67.66 & 211. & 5.35 & 59.49 & 82.66 & 242. & 5.01 & 63.85 & 93.59 \\
\hline 173. & 5.70 & 56.01 & 68.36 & 212. & 5.34 & 59.59 & 83.20 & 243. & 5.00 & 63.95 & 93.75 \\
\hline 174. & 5.69 & 56.10 & 68.91 & 213. & 5.33 & 59.69 & 83.67 & 244. & 4.96 & 64.82 & 93.98 \\
\hline 175. & 5.68 & 56.20 & 69.53 & 214. & 5.32 & 59.78 & 84.30 & 245 & 4.95 & 65.69 & 94.30 \\
\hline 176. & 5.67 & 56.30 & 70.00 & 215. & 5.31 & 59.98 & 84.84 & 246. & 4.94 & 66.08 & 94.53 \\
\hline 177. & 5.66 & 56.39 & 70.55 & 216. & 5.29 & 60.27 & 85.31 & 247. & 4.93 & 66.18 & 94.77 \\
\hline 178. & 5.65 & 56.49 & 71.09 & 217. & 5.28 & 60.46 & 85.78 & 248. & 4.92 & 66.27 & 95.00 \\
\hline 179. & 5.63 & 56.59 & 71.33 & 218. & 5.26 & 60.56 & 86.25 & 249 . & 4.91 & 66.47 & 95.23 \\
\hline 180. & 5.62 & 56.68 & 71.56 & 219. & 5.25 & 60.66 & 86.80 & 250 . & 4.90 & 66.66 & 95.47 \\
\hline 181. & 5.59 & 56.78 & 71.95 & 220. & 5.24 & 60.75 & 87.03 & 251 . & 4.89 & 66.86 & 95.70 \\
\hline 182. & 5.58 & 56.88 & 72.89 & 221. & 5.23 & 60.85 & 87.34 & 252. & 4.87 & 67.05 & 96.02 \\
\hline 183. & 5.57 & 56.97 & 73.75 & 222. & 5.22 & 60.95 & 87.66 & 253. & 4.86 & 67.44 & 96.25 \\
\hline 184. & 5.56 & 57.26 & 74.61 & 223. & 5.21 & 61.04 & 87.97 & 254 & 4.85 & 68.41 & 96.41 \\
\hline 185. & 5.55 & 57.46 & 75.16 & 224. & 5.20 & 61.14 & 88.28 & 255 & 4.83 & 68.79 & 96.56 \\
\hline 186. & 5.54 & 57.65 & 75.86 & 225 . & 5.19 & 61.24 & 88.59 & 256. & 4.82 & 68.99 & 96.72 \\
\hline 187. & 5.53 & 57.75 & 76.72 & 226. & 5.19 & 61.43 & 89.06 & 257. & 4.81 & 69.37 & 96.88 \\
\hline 188. & 5.52 & 57.85 & 77.42 & 227. & 5.19 & 61.53 & 89.61 & 258. & 4.80 & 69.47 & 97.03 \\
\hline 189. & 5.51 & 57.94 & 77.73 & 228. & 5.18 & 61.62 & 90.00 & 259. & 4.79 & 69.86 & 97.27 \\
\hline 190. & 5.49 & 58.04 & 78.20 & 229. & 5.17 & 61.72 & 90.31 & 260. & 4.78 & 69.96 & 97.58 \\
\hline 191. & 5.48 & 58.14 & 78.67 & 230 . & 5.16 & 61.82 & 90.63 & 261. & 4.77 & 70.15 & 97.81 \\
\hline 192. & 4.76 & 70.25 & 97.97 & 266. & 4.69 & 71.70 & 98.75 & 270 . & 4.53 & 73.44 & 99.53 \\
\hline 193. & 4.75 & 70.63 & 98.13 & 267. & 4.65 & 71.89 & 98.91 & 271. & 4.51 & 76.25 & 99.69 \\
\hline 194. & 4.73 & 70.73 & 98.28 & 268. & 4.64 & 71.99 & 99.06 & 272. & 4.43 & 76.74 & 99.84 \\
\hline 195. & 4.72 & 71.31 & 98.52 & 269. & 4.62 & 72.28 & 99.30 & 273. & 4.22 & 86.13 & 100 \\
\hline
\end{tabular}

Table (2) results reveal highest raw score of (10.39) with T-score (29.85) and percentile score (0.16) and lowest raw score of (4.22) with T-score (86.13) and percentile score (100). It is clear that as raw score decreases $\mathrm{T}$ score and percentile score increase due that time is improving while reducing. 
Table (3)

T standard score and percentile standard score for agility test for applicant students for addition in Qaliubiya governorate sport school

\begin{tabular}{|c|c|c|c|c|c|c|c|c|c|c|c|}
\hline No & $\begin{array}{l}\text { Raw } \\
\text { score }\end{array}$ & $\begin{array}{c}\mathrm{T} \\
\text { score }\end{array}$ & $\begin{array}{c}\text { Percentile } \\
\text { score }\end{array}$ & No & $\begin{array}{l}\text { Raw } \\
\text { score }\end{array}$ & $\begin{array}{c}\mathrm{T} \\
\text { score }\end{array}$ & $\begin{array}{c}\text { Percentile } \\
\text { score }\end{array}$ & No & $\begin{array}{l}\text { Raw } \\
\text { score }\end{array}$ & $\begin{array}{c}\mathrm{T} \\
\text { score }\end{array}$ & $\begin{array}{c}\text { Percentile } \\
\text { score }\end{array}$ \\
\hline 1. & 11.78 & 25.68 & 0.23 & 14. & 10.38 & 36.53 & 5.16 & 27. & 9.79 & 39.06 & 11.8 \\
\hline 2. & 11.25 & 29.99 & 0.47 & 15. & 10.35 & 36.68 & 5.63 & 28. & 9.78 & 39.21 & 12.34 \\
\hline 3. & 11.13 & 31.18 & 0.7 & 16. & 10.21 & 36.83 & 6.02 & 29. & 9.77 & 39.36 & 12.89 \\
\hline 4. & 11.09 & 31.33 & 0.94 & 17. & 10.14 & 36.98 & 6.56 & 30. & 9.76 & 39.51 & 13.83 \\
\hline 5. & 10.74 & 31.93 & 1.17 & 18. & 10.12 & 37.28 & 7.34 & 31. & 9.74 & 39.8 & 15.16 \\
\hline 6. & 10.72 & 32.82 & 1.41 & 19. & 10.06 & 37.43 & 7.97 & 32. & 9.73 & 40.55 & 16.17 \\
\hline 7. & 10.7 & 32.97 & 1.95 & 20. & 10.04 & 37.72 & 8.13 & 33. & 9.71 & 40.7 & 16.8 \\
\hline 8. & 10.68 & 34.01 & 2.5 & 21. & 10.03 & 37.87 & 8.52 & 34. & 9.7 & 40.84 & 17.19 \\
\hline 9. & 10.59 & 34.16 & 2.73 & 22. & 10.00 & 38.02 & 8.98 & 35. & 9.65 & 41.14 & 17.97 \\
\hline 10. & 10.51 & 34.45 & 3.75 & 23. & 9.87 & 38.17 & 9.22 & 36. & 9.64 & 41.29 & 18.75 \\
\hline 11. & 10.51 & 34.9 & 3.91 & 24. & 9.84 & 38.32 & 9.77 & 37. & 9.63 & 41.74 & 19.69 \\
\hline 12. & 10.5 & 35.2 & 4.14 & 25. & 9.83 & 38.47 & 10.55 & 38. & 9.62 & 41.88 & 20.63 \\
\hline 13. & 10.46 & 36.24 & 4.53 & 26. & 9.82 & 38.91 & 11.17 & 39. & 9.61 & 42.18 & 21.02 \\
\hline 14. & 9.6 & 42.33 & 21.56 & 70. & 9.19 & 46.94 & 41.33 & 100. & 8.81 & 52.29 & 64.22 \\
\hline 15. & 9.59 & 42.48 & 22.27 & 71. & 9.18 & 47.09 & 42.03 & 101. & 8.8 & 52.44 & 64.92 \\
\hline 16. & 9.57 & 42.63 & 22.97 & 72. & 9.16 & 47.23 & 42.34 & 102. & 8.79 & 52.59 & 65.55 \\
\hline 17. & 9.55 & 42.78 & 23.59 & 73. & 9.15 & 47.38 & 43.2 & 103. & 8.78 & 52.73 & 66.48 \\
\hline 18. & 9.54 & 42.92 & 24.45 & 74. & 9.14 & 47.53 & 44.22 & 104. & 8.77 & 53.03 & 67.42 \\
\hline 19. & 9.53 & 43.07 & 25.23 & 75. & 9.12 & 47.68 & 44.77 & 105. & 8.76 & 53.18 & 67.89 \\
\hline 20. & 9.52 & 43.22 & 25.78 & 76. & 9.11 & 47.83 & 45 & 106. & 8.75 & 53.33 & 68.44 \\
\hline 21. & 9.49 & 43.37 & 26.56 & 77. & 9.1 & 47.98 & 45.55 & 107. & 8.74 & 53.63 & 68.98 \\
\hline 22. & 9.48 & 43.52 & 27.27 & 78. & 9.09 & 48.13 & 46.09 & 108. & 8.73 & 53.77 & 69.3 \\
\hline 23. & 9.47 & 43.67 & 27.66 & 79. & 9.07 & 48.28 & 46.33 & 109. & 8.72 & 53.92 & 70 \\
\hline 24. & 9.44 & 43.82 & 28.28 & 80. & 9.06 & 48.42 & 47.19 & 110. & 8.71 & 54.07 & 70.78 \\
\hline 25. & 9.42 & 43.96 & 29.61 & 81. & 9.05 & 48.57 & 48.59 & 111. & 8.7 & 54.37 & 71.33 \\
\hline 26. & 9.41 & 44.11 & 30.78 & 82. & 9.04 & 48.87 & 49.69 & 112. & 8.69 & 54.52 & 71.64 \\
\hline 27. & 9.4 & 44.26 & 31.72 & 83. & 9.03 & 49.02 & 50.23 & 113. & 8.67 & 54.81 & 71.95 \\
\hline 28. & 9.39 & 44.41 & 32.66 & 84. & 9.02 & 49.32 & 50.86 & 114. & 8.66 & 54.96 & 72.27 \\
\hline 29. & 9.38 & 44.56 & 32.97 & 85. & 9.01 & 49.46 & 52.03 & 115. & 8.65 & 55.11 & 73.28 \\
\hline 30. & 9.37 & 44.71 & 33.2 & 86. & 8.99 & 49.76 & 53.67 & 116. & 8.64 & 55.41 & 74.38 \\
\hline 31. & 9.35 & 44.86 & 33.52 & 87. & 8.97 & 49.91 & 55.47 & 117. & 8.63 & 55.56 & 74.61 \\
\hline 32. & 9.34 & 45.01 & 33.75 & 88. & 8.96 & 50.06 & 56.56 & 118. & 8.62 & 55.71 & 74.92 \\
\hline 33. & 9.33 & 45.15 & 34.45 & 89. & 8.95 & 50.21 & 56.95 & 119. & 8.61 & 55.86 & 75.16 \\
\hline 34. & 9.32 & 45.3 & 35.16 & 90. & 8.94 & 50.36 & 57.34 & 120. & 8.6 & 56 & 75.47 \\
\hline 35. & 9.31 & 45.45 & 35.39 & 91. & 8.93 & 50.5 & 57.89 & 121. & 8.59 & 56.15 & 75.94 \\
\hline 36. & 9.3 & 45.6 & 35.7 & 92. & 8.92 & 50.8 & 58.98 & 122. & 8.58 & 56.45 & 76.56 \\
\hline 37. & 9.28 & 45.75 & 36.48 & 93. & 8.9 & 51.1 & 60.23 & 123. & 8.57 & 56.6 & 77.58 \\
\hline 38. & 9.27 & 45.9 & 37.5 & 94. & 8.89 & 51.25 & 61.02 & 124. & 8.56 & 56.75 & 78.28 \\
\hline
\end{tabular}


Follow Table (3)

T standard score and percentile standard score for agility test for applicant students for addition in Qaliubiya governorate sport school

\begin{tabular}{|c|c|c|c|c|c|c|c|c|c|c|c|}
\hline No & $\begin{array}{l}\text { Raw } \\
\text { score }\end{array}$ & $\begin{array}{c}\mathrm{T} \\
\text { score }\end{array}$ & $\begin{array}{c}\text { Percentile } \\
\text { score }\end{array}$ & No & $\begin{array}{l}\text { Raw } \\
\text { score }\end{array}$ & $\begin{array}{c}\mathrm{T} \\
\text { score }\end{array}$ & $\begin{array}{c}\text { Percentile } \\
\text { score }\end{array}$ & No & $\begin{array}{l}\text { Raw } \\
\text { score }\end{array}$ & $\begin{array}{c}\mathrm{T} \\
\text { score }\end{array}$ & $\begin{array}{c}\text { Percentile } \\
\text { score }\end{array}$ \\
\hline 39. & 9.26 & 46.05 & 38.52 & 95. & 8.87 & 51.4 & 61.88 & 125. & 8.55 & 56.9 & 78.59 \\
\hline 40. & 9.24 & 46.34 & 39.22 & 96. & 8.86 & 51.55 & 62.58 & 126. & 8.54 & 57.04 & 79.06 \\
\hline 41. & 9.23 & 46.49 & 39.61 & 97. & 8.84 & 51.69 & 62.97 & 127. & 8.53 & 57.19 & 79.53 \\
\hline 42. & 9.21 & 46.64 & 40 & 98. & 8.83 & 51.84 & 63.44 & 128. & 8.52 & 57.49 & 79.84 \\
\hline 43. & 9.2 & 46.79 & 40.39 & 99. & 8.82 & 51.99 & 63.75 & 129. & 8.51 & 57.94 & 80.00 \\
\hline 44. & 8.5 & 58.08 & 80.47 & 146. & 8.3 & 61.8 & 89.06 & 162. & 8.05 & 68.93 & 95.23 \\
\hline 45. & 8.49 & 58.23 & 81.02 & 147. & 8.25 & 61.95 & 89.69 & 163. & 8.04 & 71.02 & 95.86 \\
\hline 46. & 8.48 & 58.68 & 81.56 & 148. & 8.23 & 62.25 & 90.16 & 164. & 8.03 & 71.46 & 96.33 \\
\hline 47. & 8.47 & 58.83 & 82.42 & 149. & 8.22 & 62.4 & 90.39 & 165. & 8.01 & 72.65 & 96.56 \\
\hline 48. & 8.46 & 58.98 & 83.13 & 150. & 8.21 & 62.54 & 90.63 & 166. & 7.94 & 73.25 & 96.72 \\
\hline 49. & 8.45 & 59.13 & 83.52 & 151. & 8.2 & 62.69 & 90.78 & 167. & 7.92 & 73.39 & 96.95 \\
\hline 50. & 8.44 & 59.42 & 83.91 & 152. & 8.19 & 63.14 & 91.17 & 168. & 7.89 & 73.39 & 96.95 \\
\hline 51. & 8.43 & 59.72 & 84.38 & 153. & 8.16 & 63.29 & 91.72 & 169. & 7.87 & 74.58 & 97.19 \\
\hline 52. & 8.42 & 59.87 & 84.69 & 154. & 8.15 & 63.44 & 92.03 & 170. & 7.86 & 75.92 & 97.42 \\
\hline 53. & 8.41 & 60.02 & 84.84 & 155. & 8.14 & 63.88 & 92.27 & 171. & 7.79 & 76.22 & 97.73 \\
\hline 54. & 8.39 & 60.17 & 85.16 & 156. & 8.13 & 65.81 & 92.89 & 172. & 7.78 & 76.52 & 98.13 \\
\hline 55. & 8.38 & 60.31 & 86.25 & 157. & 8.12 & 66.26 & 93.52 & 173. & 7.72 & 76.81 & 98.75 \\
\hline 56. & 8.35 & 60.46 & 87.34 & 158. & 8.11 & 66.41 & 93.83 & 174. & 7.68 & 82.01 & 99.38 \\
\hline 57. & 8.34 & 60.61 & 87.81 & 159. & 8.09 & 66.71 & 94.14 & 175. & 7.67 & 82.61 & 99.69 \\
\hline 58. & 8.32 & 61.35 & 88.28 & 160. & 8.08 & 67.6 & 94.38 & 176. & 7.59 & 84.39 & 99.84 \\
\hline 59. & 8.31 & 61.5 & 88.67 & 161. & 8.06 & 67.89 & 94.69 & 177. & 7.3 & 92.27 & 100 \\
\hline
\end{tabular}

Table (3) results reveal highest raw score of (11.78) with T-score (25.60) and percentile score $(0.23)$ and lowest raw score of (7.30) with T-score (92.27) and percentile score (100). It is clear that as raw score decreases $\mathrm{T}$ score and percentile score increase due that time is improving while reducing. 
Table (4)

$T$ standard score and percentile standard score for jump test for applicant students for addition in Qaliubiya governorate sport school

\begin{tabular}{|c|c|c|c|c|c|c|c|c|c|c|c|}
\hline No & $\begin{array}{l}\text { Raw } \\
\text { score }\end{array}$ & $\begin{array}{c}\mathrm{T} \\
\text { score }\end{array}$ & $\begin{array}{c}\text { Percentile } \\
\text { score }\end{array}$ & No & $\begin{array}{l}\text { Raw } \\
\text { score }\end{array}$ & $\begin{array}{c}\mathrm{T} \\
\text { score }\end{array}$ & $\begin{array}{c}\text { Percentile } \\
\text { score }\end{array}$ & No & $\begin{array}{l}\text { Raw } \\
\text { score }\end{array}$ & $\begin{array}{c}\mathrm{T} \\
\text { score }\end{array}$ & $\begin{array}{c}\text { Percentile } \\
\text { score }\end{array}$ \\
\hline 1. & 0.90 & 14.91 & 0.23 & 22. & 1.53 & 45.97 & 34.38 & 43. & 1.79 & 58.78 & 81.02 \\
\hline 2. & 1.00 & 19.84 & 0.55 & 23. & 1.55 & 46.95 & 37.03 & 44. & 1.80 & 59.28 & 84.3 \\
\hline 3. & 1.10 & 24.77 & 1.17 & 24. & 1.56 & 47.44 & 39.84 & 45. & 1.81 & 59.77 & 87.5 \\
\hline 4. & 1.15 & 27.23 & 1.8 & 25. & 1.57 & 47.94 & 40.16 & 46. & 1.82 & 60.26 & 87.81 \\
\hline 5. & 1.20 & 29.7 & 2.66 & 26. & 1.58 & 48.43 & 40.31 & 47. & 1.84 & 61.25 & 88.13 \\
\hline 6. & 1.22 & 30.68 & 3.44 & 27. & 1.59 & 48.92 & 40.47 & 48. & 1.85 & 61.74 & 89.77 \\
\hline 7. & 1.25 & 32.16 & 4.38 & 28. & 1.60 & 49.42 & 45.78 & 49. & 1.89 & 63.71 & 91.41 \\
\hline 8. & 1.30 & 34.63 & 6.64 & 29. & 1.61 & 49.91 & 51.17 & 50. & 1.90 & 64.21 & 92.97 \\
\hline 9. & 1.33 & 36.11 & 8.13 & 30. & 1.62 & 50.4 & 51.8 & 51. & 1.92 & 65.19 & 94.77 \\
\hline 10. & 1.34 & 36.6 & 8.28 & 31. & 1.63 & 50.9 & 52.34 & 52. & 1.93 & 65.68 & 95.16 \\
\hline 11. & 1.35 & 37.09 & 9.69 & 32. & 1.64 & 51.39 & 52.5 & 53. & 1.94 & 66.18 & 95.31 \\
\hline 12. & 1.38 & 38.57 & 11.09 & 33. & 1.65 & 51.88 & 57.03 & 54. & 1.95 & 66.67 & 95.78 \\
\hline 13. & 1.40 & 39.56 & 13.83 & 34. & 1.66 & 52.37 & 61.64 & 55. & 1.96 & 67.16 & 96.25 \\
\hline 14. & 1.43 & 41.04 & 16.56 & 35. & 1.67 & 52.87 & 61.95 & 56. & 1.97 & 67.66 & 96.41 \\
\hline 15. & 1.45 & 42.02 & 18.83 & 36. & 1.68 & 53.36 & 62.66 & 57. & 2.00 & 69.14 & 97.66 \\
\hline 16. & 1.46 & 42.51 & 21.09 & 37. & 1.70 & 54.35 & 67.03 & 58. & 2.05 & 71.6 & 99.06 \\
\hline 17. & 1.47 & 43.01 & 21.33 & 38. & 1.72 & 55.33 & 71.02 & 59. & 2.06 & 72.09 & 99.38 \\
\hline 18. & 1.48 & 43.5 & 21.64 & 39. & 1.73 & 55.82 & 71.48 & 60. & 2.10 & 74.07 & 99.53 \\
\hline 19. & 1.50 & 44.49 & 27.19 & 40. & 1.74 & 56.32 & 72.03 & 61. & 2.15 & 76.53 & 99.69 \\
\hline 20. & 1.51 & 44.98 & 32.97 & 41. & 1.76 & 57.3 & 80.08 & 62. & 2.25 & 81.46 & 99.84 \\
\hline 21. & 1.52 & 45.47 & 33.83 & 42. & 1.78 & 58.29 & 80.7 & 63. & 2.70 & 83.64 & 100 \\
\hline
\end{tabular}

Table (4) results reveal highest raw score of (2.70) with T-score (83.64) and percentile score (100) and lowest raw score of $(0.90)$ with
T-score (14.91) and percentile score $(0.32)$. It is clear that as raw score increases $\mathrm{T}$ score and percentile score increase. 
Table (5)

T standard score and percentile standard score for pushing test for applicant students for addition in Qaliubiya governorate sport school

\begin{tabular}{|c|c|c|c|c|c|c|c|c|c|c|c|}
\hline No & $\begin{array}{l}\text { Raw } \\
\text { score }\end{array}$ & $\begin{array}{c}\mathrm{T} \\
\text { score }\end{array}$ & $\begin{array}{c}\text { Percentile } \\
\text { score }\end{array}$ & No & $\begin{array}{l}\text { Raw } \\
\text { score }\end{array}$ & $\begin{array}{c}\mathrm{T} \\
\text { score }\end{array}$ & $\begin{array}{l}\text { Percentile } \\
\text { score }\end{array}$ & No & $\begin{array}{l}\text { Raw } \\
\text { score }\end{array}$ & $\begin{array}{c}\mathrm{T} \\
\text { score }\end{array}$ & $\begin{array}{c}\text { Percentile } \\
\text { score }\end{array}$ \\
\hline 1. & 1.2 & 36.25 & 0.16 & 31. & 2.92 & 45.65 & 54.84 & 61. & 5.4 & 59.21 & 75.31 \\
\hline 2. & 1.5 & 37.89 & 0.31 & 32. & 2.95 & 45.81 & 55 & 62. & 5.5 & 59.75 & 76.02 \\
\hline 3. & 1.55 & 38.16 & 0.7 & 33. & 3 & 46.09 & 56.64 & 63. & 5.6 & 60.3 & 77.19 \\
\hline 4. & 1.6 & 38.43 & 1.41 & 34. & 3.05 & 46.36 & 59.06 & 64. & 5.7 & 60.85 & 78.05 \\
\hline 5. & 1.65 & 38.71 & 2.03 & 35. & 3.1 & 46.63 & 61.33 & 65. & 5.75 & 61.12 & 78.44 \\
\hline 6. & 1.8 & 39.53 & 2.5 & 36. & 3.15 & 46.91 & 63.05 & 66. & 5.8 & 61.39 & 79.38 \\
\hline 7. & 1.85 & 39.8 & 3.05 & 37. & 3.2 & 47.18 & 63.67 & 67. & 5.9 & 61.94 & 80.63 \\
\hline 8. & 1.9 & 40.07 & 4.22 & 38. & 3.25 & 47.45 & 64.3 & 68. & 5.95 & 62.21 & 81.09 \\
\hline 9. & 1.95 & 40.35 & 5.31 & 39. & 3.35 & 48 & 65.08 & 69. & 6 & 62.49 & 82.03 \\
\hline 10. & 2 & 40.62 & 7.58 & 40. & 3.4 & 48.27 & 65.31 & 70. & 6.05 & 62.76 & 83.13 \\
\hline 11. & 2.05 & 40.89 & 10.55 & 41. & 3.6 & 49.37 & 65.55 & 71. & 6.1 & 63.03 & 83.75 \\
\hline 12. & 2.1 & 41.17 & 13.2 & 42. & 3.7 & 49.91 & 65.78 & 72. & 6.15 & 63.31 & 84.38 \\
\hline 13. & 2.15 & 41.44 & 15.08 & 43. & 3.75 & 50.19 & 65.94 & 73. & 6.2 & 63.58 & 84.92 \\
\hline 14. & 2.2 & 41.71 & 16.02 & 44. & 3.8 & 50.46 & 66.33 & 74. & 6.25 & 63.85 & 85.63 \\
\hline 15. & 2.25 & 41.99 & 17.97 & 45. & 3.9 & 51.01 & 66.88 & 75. & 6.3 & 64.13 & 86.64 \\
\hline 16. & 2.3 & 42.26 & 20.63 & 46. & 4 & 51.55 & 67.27 & 76. & 6.35 & 64.4 & 87.42 \\
\hline 17. & 2.33 & 42.43 & 22.19 & 47. & 4.2 & 52.65 & 67.66 & 77. & 6.4 & 64.67 & 87.81 \\
\hline 18. & 2.35 & 42.53 & 22.58 & 48. & 4.3 & 53.19 & 67.97 & 78. & 6.45 & 64.95 & 88.28 \\
\hline 19. & 2.4 & 42.81 & 25.78 & 49. & 4.4 & 53.74 & 68.13 & 79. & 6.5 & 65.22 & 88.98 \\
\hline 20. & 2.45 & 43.08 & 29.3 & 50. & 4.5 & 54.29 & 68.52 & 80. & 6.55 & 65.49 & 89.61 \\
\hline 21. & 2.5 & 43.35 & 33.59 & 51. & 4.7 & 55.38 & 69.06 & 81. & 6.6 & 65.77 & 90.23 \\
\hline 22. & 2.53 & 43.52 & 37.34 & 52. & 4.8 & 55.93 & 69.84 & 82. & 6.65 & 66.04 & 90.86 \\
\hline 23. & 2.55 & 43.63 & 37.89 & 53. & 4.9 & 56.47 & 70.7 & 83. & 6.7 & 66.31 & 91.25 \\
\hline 24. & 2.6 & 43.9 & 39.92 & 54. & 5 & 57.02 & 71.33 & 84. & 6.8 & 66.86 & 92.11 \\
\hline 25. & 2.65 & 44.17 & 41.72 & 55. & 5.05 & 57.29 & 71.8 & 85. & 6.9 & 67.41 & 93.05 \\
\hline 26. & 2.7 & 44.45 & 43.2 & 56. & 5.1 & 57.57 & 72.34 & 86. & 7 & 67.95 & 93.75 \\
\hline 27. & 2.75 & 44.72 & 46.02 & 57. & 5.15 & 57.84 & 72.81 & 87. & 7.05 & 68.23 & 94.53 \\
\hline 28. & 2.8 & 44.99 & 49.45 & 58. & 5.2 & 58.11 & 73.28 & 88. & 7.1 & 68.5 & 95.08 \\
\hline 29. & 2.85 & 45.27 & 51.95 & 59. & 5.25 & 58.39 & 73.91 & 89. & 7.2 & 69.05 & 95.31 \\
\hline 30. & 2.9 & 45.54 & 53.67 & 60. & 5.3 & 58.66 & 74.69 & 90. & 7.25 & 69.32 & 95.47 \\
\hline 31. & 7.3 & 69.59 & 95.94 & 96. & 7.6 & 71.23 & 98.2 & 101. & 8.6 & 76.7 & 99.53 \\
\hline 32. & 7.35 & 69.87 & 96.48 & 97. & 7.7 & 71.78 & 98.44 & 102. & 9 & 78.89 & 99.69 \\
\hline 33. & 7.4 & 70.14 & 96.8 & 98. & 7.8 & 72.33 & 98.75 & 103. & 9.5 & 81.62 & 99.84 \\
\hline 34. & 7.5 & 70.69 & 97.34 & 99. & 7.85 & 72.6 & 99.06 & 104. & 9.9 & 83.81 & 100 \\
\hline 35. & 7.55 & 70.96 & 97.89 & 100. & 8.4 & 75.61 & 99.3 & 105. & & & \\
\hline
\end{tabular}


Table (5) results reveal highest raw score of (9.9) with T-score (83.81) and percentile score (100) and lowest raw score of (1.2) with T-score
(36.25) and percentile score (0.16). It is clear that as raw score increases $\mathrm{T}$ score and percentile score increase.

\section{Table (6)}

$T$ standard score and percentile standard score for abdomen test for applicant students for addition in Qaliubiya governorate sport school

\begin{tabular}{l|c|c|c|c|c|c|c|c|c|c|c}
\hline \hline No & $\begin{array}{c}\text { Raw } \\
\text { score }\end{array}$ & $\begin{array}{c}\text { T } \\
\text { score }\end{array}$ & $\begin{array}{c}\text { Percentile } \\
\text { score }\end{array}$ & No & $\begin{array}{c}\text { Raw } \\
\text { score }\end{array}$ & $\begin{array}{c}\text { T } \\
\text { score }\end{array}$ & $\begin{array}{c}\text { Percentile } \\
\text { score }\end{array}$ & No & $\begin{array}{c}\text { Raw } \\
\text { score }\end{array}$ & $\begin{array}{c}\text { T } \\
\text { score }\end{array}$ & $\begin{array}{c}\text { Percentile } \\
\text { score }\end{array}$ \\
\hline \hline 1. & 12 & 18.56 & 0.16 & 10. & 21 & 42.2 & 21.02 & 19. & 30 & 65.84 & 93.91 \\
\hline 2. & 13 & 21.19 & 0.39 & 11. & 22 & 44.83 & 30.31 & 20. & 31 & 68.47 & 96.72 \\
\hline 3. & 14 & 23.81 & 1.02 & 12. & 23 & 47.46 & 41.25 & 21. & 32 & 71.1 & 97.89 \\
\hline 4. & 15 & 26.44 & 1.56 & 13. & 24 & 50.08 & 52.89 & 22. & 33 & 73.72 & 98.67 \\
\hline 5. & 16 & 29.07 & 2.34 & 14. & 25 & 52.71 & 62.66 & 23. & 34 & 76.35 & 99.38 \\
\hline 6. & 17 & 31.7 & 3.2 & 15. & 26 & 55.34 & 71.09 & 24. & 35 & 78.98 & 99.84 \\
\hline 7. & 18 & 34.32 & 4.84 & 16. & 27 & 57.96 & 78.98 & 25. & 36 & 81.6 & 100 \\
\hline 8. & 19 & 36.95 & 8.2 & 17. & 28 & 60.59 & 85.31 & & & & \\
\hline 9. & 20 & 39.58 & 13.44 & 18. & 29 & 63.22 & 90 & & & & \\
\hline \hline
\end{tabular}

Table (6) results reveal highest raw score of (36) with T-score (81.60) and percentile score (100) and lowest raw score of (12) with T-score
(18.56) and percentile score (0.16). It is clear that as raw score increases $\mathrm{T}$ score and percentile score increase.

Table (7)

Correlation coefficient between Body Mass Index (BMI) and the $T$-scores for tests under study $(n=640)$

\begin{tabular}{c|c|c|c|c|c}
\hline \hline Variable & \multicolumn{5}{|c}{ Correlation coefficient (R) with } \\
\cline { 2 - 6 } & Speed & Agility & $\begin{array}{c}\text { Legs } \\
\text { power }\end{array}$ & $\begin{array}{c}\text { Arms } \\
\text { Power }\end{array}$ & $\begin{array}{c}\text { Abdomen } \\
\text { strength }\end{array}$ \\
\hline \hline BMI & $0.171^{*}$ & $0.139^{*}$ & $-0.228^{*}$ & $0.123^{*}$ & $0.112^{*}$ \\
\hline \hline
\end{tabular}

* Significant at 0.05 level $(\mathrm{R}$ significant $=0.078)$

Table (7) results reveal availability of statistically significant positive correlation between BMI and (speed, agility and arms power) and statistically significant negative correlation between BMI and (legs power and abdomen strength)

Assiut Journal For Sport Science Arts 
Table (8)

Linear regression for speed on body mass index $(n=640)$

\begin{tabular}{|c|c|c|c|c|c|c|}
\hline variables & $\begin{array}{c}\text { Linear } \\
\text { regression } \\
\text { coefficient } \\
\text { (B) }\end{array}$ & $\begin{array}{c}\text { Standard } \\
\text { error }\end{array}$ & $\mathbf{D F}$ & $\mathbf{T}$ & $\mathbf{F}$ & $\begin{array}{c}\text { Contribution } \\
\%\left(\mathbf{R}^{2}\right)\end{array}$ \\
\hline $\begin{array}{l}\text { Constant } \\
\text { BMI }\end{array}$ & $\begin{array}{c}40.873 \\
0.460\end{array}$ & $\begin{array}{l}2.223 \\
0.110\end{array}$ & $\overline{1}$ & $\begin{array}{l}\frac{18.338}{4.171} \\
\end{array}$ & 17.396 & 0.025 \\
\hline
\end{tabular}

Table (8) results reveal the following prediction formula:

Speed $=40.873+0.460 \times \mathrm{BMI}$

Table (9)

Linear regression for agility on body mass index $(n=640)$

\begin{tabular}{l|c|c|c|c|c|c}
\hline \hline variables & $\begin{array}{c}\text { Linear } \\
\text { regression } \\
\text { coefficient } \\
(\mathbf{B})\end{array}$ & $\begin{array}{c}\text { Standard } \\
\text { error }\end{array}$ & $\mathbf{D F}$ & $\mathbf{T}$ & $\mathbf{F}$ & $\begin{array}{c}\text { Contribution } \\
\boldsymbol{\%}\left(\mathbf{R}^{\mathbf{t}}\right)\end{array}$ \\
\hline Constant & 41.536 & 2.227 & $\mathbf{1}$ & $\frac{18.651}{1}$ & 14.903 & \\
\hline BMI & 0.426 & 0.110 & & 3.860 & & 0.023 \\
\hline \hline
\end{tabular}

Table (9) results reveal the following prediction formula:

Speed $=41.536+0.426 \times$ BMI

Table (10)

Linear regression for legs power on body mass index $(n=640)$

\begin{tabular}{|c|c|c|c|c|c|c|}
\hline variables & $\begin{array}{c}\text { Linear } \\
\text { regression } \\
\text { coefficient } \\
\text { (B) }\end{array}$ & $\begin{array}{c}\text { Standard } \\
\text { error }\end{array}$ & $\overline{\mathrm{DF}}$ & $\mathbf{T}$ & $\mathbf{F}$ & $\begin{array}{c}\text { Contribution } \\
\%\left(\mathbf{R}^{2}\right)\end{array}$ \\
\hline $\begin{array}{l}\text { Constant } \\
\text { BMI }\end{array}$ & $\begin{array}{l}62.329 \\
-0.621\end{array}$ & $\begin{array}{l}2.198 \\
0.109\end{array}$ & T & $\begin{array}{l}28.361 \\
5.698\end{array}$ & 32.437 & 0.048 \\
\hline
\end{tabular}

Table (10) results reveal the following prediction formula:

Speed $=62.329-0.621 \times$ BMI

Table (11)

Linear regression for arms power on body mass index $(n=640)$

\begin{tabular}{l|c|c|c|c|c|c|}
\hline \hline variables & $\begin{array}{c}\text { Linear } \\
\text { regression } \\
\text { coefficient } \\
(\mathbf{B})\end{array}$ & $\begin{array}{c}\text { Standard } \\
\text { error }\end{array}$ & $\mathbf{D F}$ & $\mathbf{T}$ & $\mathbf{F}$ & $\begin{array}{c}\text { Contribution } \\
\mathbf{\%}\left(\mathbf{R}^{\mathbf{2}}\right)\end{array}$ \\
\hline Constant & 46.716 & 2.249 & \multirow{1}{*}{$\mathbf{1}$} & 20.771 & 2.200 & \\
\cline { 1 - 5 } BMI & 0.165 & 0.111 & & 1.483 & & 0.003 \\
\hline \hline
\end{tabular}

Table (8) results reveal the following prediction formula:

Speed $=46.716+0.165 \times \mathrm{BMI}$

Assiut Journal For Sport Science Arts 
Table (12)

Linear regression for abdomen muscles strength on body mass index $(n=640)$

\begin{tabular}{l|c|c|c|c|c|c}
\hline \hline variables & $\begin{array}{c}\text { Linear } \\
\text { regression } \\
\text { coefficient } \\
(\mathbf{B})\end{array}$ & $\begin{array}{c}\text { Standard } \\
\text { error }\end{array}$ & $\mathbf{D F}$ & $\mathbf{T}$ & $\mathbf{F}$ & $\begin{array}{c}\text { Contribution } \\
\mathbf{\%}\left(\mathbf{R}^{\mathbf{2}}\right)\end{array}$ \\
\hline \hline Constant & 55.854 & 2.241 & $\mathbf{1}$ & 24.928 & 7.0443 & \\
\cline { 1 - 2 } \cline { 5 - 7 } & -0.295 & 0.111 & & 2.654 & & 0.011 \\
\hline \hline
\end{tabular}

Table (8) results reveal the following prediction formula:

Speed $=55.854-0.290 \times$ BMI

Conclusions:

In light of study aim and in limits of tests applied and statistical analysis results the following concluded:

- $\quad \mathrm{T}$ and percentile scores set for speed, agility, arms power, legs power and abdomen muscles strength tests - Prediction formula was set for physical tests by knowing BMI

\section{Recommendations:}

- Using standard score table concluded by the study in evaluating student apply for sports preparatory schools in Arab republic of Egypt.

- Doing similar study to direct the students to specific activity

- Archiving admission tests results in sports preparatory schools and periodically update it
- Using prediction formulas concluded by the study

- Developing standard scores for skill performances in sport schools

\section{References}

1- Abo Shady, S., Abdulsalam, A., \& Mohamed, R. (2005). Setting standard levels for some physical and physiological variables in late childhood. Sport Theories and Application, Alexandria University (53), 18-65. (in Arabic Language)

2- Allawi, A., \& Elshafee, G. (2000). Contemporary physical education curricula. Cairo. (in Arabic Language)

3- Allawi, M., \& Radwan, M. (2000). Measurement in physical education and sport pschology (2nd ed.). Cairo: Arab though house. (in Arabic Language) 
4- Hassanien, M. (1995).

Measurement in physical education in physical education and sports (3rd ed.). Cairo: Arab though house. (in Arabic Language)

5- Homs, M. (1997). The guide in physical education teaching. Alexandria: AlMaaref establishment. (in Arabic Language)

6- Maciaszek, J., \&

Osiński,, W. (2004). Height, weight, body fat, static strength and explosive power of girls aged 10-14 tested with "EUROFIT" test. Kinesiologia Slovenica, 7(1-2), 30-37. (in Arabic Language)

7- Ministry of Education. (2003). National education standards. Cairo, Egypt: Author. (in Arabic Language)

8- Mohamed, M. (2007).

Setting standard levels for some physical and skill characteristics for basketball juniors (14-16 years) (Unpublished master's thesis). Faculty of physical education, Asuit University. (in Arabic Language)

9- Radwan, M. (2006). Introduction to measurement in physical education and sport. Cairo: Book publishing center. 10- Saleh, M. (2013). Setting standard levels for Physical fitness and some anthropometric measurements for higher basic education stage in Gonain directorate (Unpublished master's thesis). Al-Nagah, Gonain, Palastine. (in Arabic Language) 11- Visscher, T., Snijder, M., \& Seidell, J. (2010). Epidemiology: definition and classification of Obesity. In P. KOPELMAN, I. CATERSON, \& W. DIETZ (Eds.), Clinical obesity in adults and children (pp. 3-14). New York: Wiley-blackwell. 